\title{
ANSIEDAD Y DEPRESIÓN EN PERSONAS CON OBESIDAD DE PARAGUAY
}

\begin{tabular}{c} 
ANXIETY AND DEPRESSION IN PEOPLE WITH OBESITY FROM PARAGUAY \\
\hline DOI: 10.22199/S07187475.2015.0001.00003 Recibido: 10 de Febrero del 2015 | Aceptado: 25 de Marzo del 2015
\end{tabular}

\author{
LETICIA REYES BRITES 1 ; JACQUELINE BENTANCUR LUSTÓ2; ANTONIO SAMANIEGO PINHO 3 \\ (UNIVERSIDAD NACIONAL DE ASUNCIÓN, Asunción, Paraguay)
}

\section{RESUMEN}

Objetivo: Determinar la relación entre depresión y ansiedad en personas con obesidad. Método: Se realizó una investigación cuantitativa, no experimental, correlacional y transversal. Participantes: 98 personas con una media de 32.43 años $(D E=10.69)$ e Índice de Masa Corporal igual o superior a 30. Instrumento: Se aplicó la adaptación de Conde y Useros del Inventario de Depresión de Beck (BDI-II) y el Inventario de Ansiedad Rasgo-Estado (STAI) de Spielberg y Díaz-Guerrero (1975). Resultados: Existieron diferencias en ansiedad-estado entre quienes recibían tratamiento de obesidad y quienes no $(t=-5.56 ; p=.000)$, en ansiedad-rasgo, entre hombres y mujeres $(t=-3.46 ; p=.001)$ y en depresión, entre hombres y mujeres $(t=-2.31 ; p=.023)$, entre los menores de 30 y los de 30 años o más $(t=2.63 ; p=.010)$, entre quienes son principal sostén de su hogar y quienes no ( $t=-2.25 ; p=.027)$, entre quienes ya tenían diagnóstico de obesidad y quienes no $(t=3.24 ; p=.002)$ y entre quienes recibían tratamiento y quienes no $(\mathrm{t}=2.21 ; \mathrm{p}=.030)$. La correlación fue positiva entre ansiedad-estado y depresión $(r=.417 ; p=.000)$, entre ansiedad-rasgo y depresión $(r=.610 ; p=.000)$ y entre ansiedad-estado y ansiedad-rasgo $(r=.544$; $\mathrm{p}=.000$ ). Conclusión: Existe relación directa entre ansiedad y depresión en personas con obesidad, aumentando 0 disminuyendo ambas en el mismo sentido.

PALABRAS CLAVE: Ansiedad, depresión, obesidad.

\section{ABSTRACT}

The aim of this paper was to determine the relationship between depression and anxiety in obese people. A quantitative, nonexperimental, correlational and cross-sectional research was conducted. The sample was composed by 98 people with an average of 32.43 years $(S D=10.69)$ and body mass index equal to or greater than 30 . Data were collected through Conde and Useros' adaptation of the Beck Depression Inventory (BDI-II) and Spielberg and Diaz-Guerrero's Inventory State-Trait Anxiety (STAI) (1975). Results show the following differences, i. in state-anxiety among those receiving treatment for obesity and those without $(t=-5.56 ; p=.000)$, ii. in trait-anxiety between men and women $(t=-3.46 ; p=0.001)$, iii. in depression between men and women ( $t=-2.31 ; p=.023)$, iv. between those under 30 and those older than $30(t=2.63 ; p=.010)$, v. between those who are home providers and those who are not $(t=-2.25 ; p=.027)$, vi. between those who already had a diagnosis of obesity and those without it $(t=3.24 ; p=.002)$ and vii. between those who received treatment and those did not $(t=2.21 ; p=.030)$. A positive correlation between state-anxiety and depression was found $(r=.417, p=.000)$, also between trait-anxiety and depression $(r=.610, p=.000)$ and between state-anxiety and trait-anxiety $(r=.544, p=.000)$. In conclusion, there is a direct relationship between anxiety and depression in people with obesity, increasing or decreasing in the same direction.

KEY WORDS: Anxiety, depression, obesity. 


\section{INTRODUCCIÓN}

La obesidad es una enfermedad crónica donde el síntoma principal es la acumulación exagerada de grasa 0 hipertrofia del tejido adiposo en el cuerpo, comprometiendo la salud del individuo (Álvarez, Monereo \& Moreno, 2000). Sus causas son diversas y en su gran mayoría evitables y prevenibles (Silvestri, 2005; Ayela, 2009).

La prevalencia mundial de la obesidad se ha multiplicado por más de dos entre 1980 y 2014 (OMS, 2014). Según la Organización Mundial de la Salud (OMS), al 2014, más de 600 millones de personas de 18 o más años eran obesos, esto es el $13 \%$ de la población adulta mundial (un 11\% de los hombres y un $15 \%$ de las mujeres).

Para poder identificar esta enfermedad existe el índice de masa corporal (IMC), es decir, el peso expresado en kilogramos divido por el cuadrado de la altura en centímetros. Según la OMS (2012) se considera sobrepeso a un IMC entre 5 y 29 $\mathrm{Kg} / \mathrm{m}^{2}$, obesidad de grado I al IMC entre 30 y $34 \mathrm{Kg} / \mathrm{m}^{2}$, obesidad de grado II al IMC entre $35-39,9 \mathrm{~kg} / \mathrm{m}^{2}$, obesidad de grado III al IMC mayor o igual a $40 \mathrm{~kg} / \mathrm{m}^{2}$ y obesidad de grado IV u obesidad extrema al IMC mayor a $50 \mathrm{~kg} / \mathrm{m}^{2}$.

La obesidad se ha asociado a una multiplicidad de síntomas comúnmente presentes, como bajo concepto de sí mismo, ansiedad y depresión (Castillo, García \& Guzmán, 2010; Calderón, Forns \& Varea, 2010a; Días \& Enríquez, 2007), las que explican en gran parte las conductas relacionadas con los trastornos de alimentación, así como también la insatisfacción corporal, potenciando de esa forma la persistencia de la obesidad (Calderón, Forns \& Varea, 2010a). A la vez, la ansiedad puede ser a su vez consecuencia de la misma acompañando a una imagen corporal distorsionada muy relacionada con desórdenes alimenticios (Bobadilla-Suárez \& López-Avila, 2014).

Las personas con obesidad utilizan el acto de degustar, masticar y triturar la comida como un recurso para bajar los niveles de ansiedad. Al pasar a ser un hábito, la persona comienza a engordar pues la única salida para compensar las situaciones estresantes de la vida como respuesta es comer en abundancia. Desde allí se puede considerar a la obesidad como el síntoma 0 la consecuencia de un problema psicológico y social (Calderón, Forns \& Varea, 2010b; Cárdenas, Ontiveros \& Villaseñor, 2006; Castillo, García \& Guzmán, 2010; Pompa, 2011; Silvestri, 2005).

En sociedades occidentales, la obesidad y el sobrepeso constituyen adicionalmente un problema moral debido a que se juzga de "gordos" o "gordas" a quienes presentan sobrepeso u obesidad cuando esto ocurre por comer mucho o cuando no pueden bajar de peso, es decir, existe un estigma social por la creencia de que estas personas tienen en sus manos el problema y la solución, y que solo por perezosas no actúan para mejorar su situación de peso. Las personas con obesidad son vistas como carentes de autocontrol y de fuerza de voluntad (Cruz Sánchez, Tuñón Pablos, Villaseñor Farías, Álvarez Gordillo \& Nigh Nielsen, 2013). Lo expuesto, puede ser motivo de ansiedad ya que entre las causas más usuales de un estado ansioso se encuentra el miedo a no alcanzar metas personales o estándares sociales (Castillo, García \& Guzmán, 2010; Silvestri, 2005).

Por otra parte, la insatisfacción de la imagen corporal regularmente producen un aislamiento social, no se animan a entablar una relación amorosa por miedo al rechazo, la burla, etc., lo cual hace que sus pensamientos y sentimientos lo lleven a realizar la sobre-ingresa de comida para evitar esas situaciones, desmotivándolo al máximo y encaminándolo a una depresión; 
en algunos produce la pérdida del apetito y en otros aumenta la ingesta (Silvestri, 2005). A este hecho, debemos sumar muchas veces la depresión como un impedimento para combatir con la obesidad. Ambos trastornos en conjunto son un arma mortal, pues la depresión hará prevalecer a la obesidad, y con ésta presente, se dificulta el cambio de actitudes y conductas y favorece las recaídas (Silvestri, 2005).

Pese a lo relevante de la temática, en Paraguay no se ha registrado alguna investigación que considere estas variables estudiándolas en personas con obesidad, por lo tanto, la presente investigación tuvo por objetivo analizar la relación entre ansiedad y depresión en personas con obesidad.

\section{MÉTODO}

\section{Tipo de estudio}

Se realizó una investigación de enfoque cuantitativo, de diseño no experimental, de alcance correlacional y transversal.

\section{Participantes}

Se incluyó a personas entre los 18 y 45 años con Índice de Masa Corporal superior a 30. Antes de iniciar la recolección de datos, se aplicó inicialmente una lista de cotejo en la que se consultó si sabían leer y escribir, si ya habían sido evaluadas anteriormente con instrumentos de ansiedad o depresión y si tenían diagnóstico previo de algún déficit de atención, así como si se encontraban en tratamiento de ansiedad 0 depresión, y consumiendo sustancias psicotrópicas, alucinógenas y/o alcohólicas; fueron excluidas aquellas personas que referían alguno de esos aspectos consultados. Se utilizó un muestreo de tipo no probabilístico e intencional. Se evaluó un total de 98 personas (Tabla 1), de los cuales el $80.6 \%$ correspondió a mujeres. El promedio de edad de toda la muestra fue 32.43 años $(D E=10.69)$. El $29.6 \%$ presentó obesidad grado I, el $38.8 \%$ presentó obesidad grado II, el $26.5 \%$ presentó obesidad grado III y el restante $5.1 \%$ presentaron obesidad grado IV. El 39\% de los participantes fueron reclutados en tres hospitales públicos, el $29 \%$ en tres facultades de una universidad pública, el $13 \%$ en dos universidades privadas, el $10 \%$ en una institución privada de tratamiento de la obesidad y el $10 \%$ en otras instituciones privadas, todas ubicadas en la ciudad de Asunción (Paraguay). El 10\% correspondiente a los participantes de la institución privada, se encontraban en tratamiento de obesidad, no habían sido evaluados previamente con los mismos instrumentos de la presente investigación y formaban parte de un programa televisivo cuyos objetivos principales eran la modificación de la conducta alimentaria, la reducción semanal del peso y el mejoramiento de la calidad de vida. Las características sociodemográficas de la muestra pueden ser observadas en la TABLA 1.

TABLA 1.

Datos socio demográficos de la muestra.

\begin{tabular}{lcc}
\hline \multicolumn{1}{c}{ Característica } & $f$ & $\%$ \\
\hline Menores de 30 años & 50 & 51 \\
30 años o más & 48 & 49 \\
Mujeres & 79 & 80.6 \\
Hombres & 19 & 19.4 \\
Soltero/a & 56 & 57.1 \\
Casado/a - Unión de Hecho & 33 & 34.7 \\
Divorciado/a - Separado/a & 6 & 6.1 \\
Viudo/a & 3 & 3.1 \\
Nivel Académico & & \\
- Primer Ciclo & 2 & 2 \\
- Segundo Ciclo & 4 & 4 \\
- Tercer Ciclo & 7 & 7 \\
- Educación Media & 25 & 26 \\
- Título Universitario & 55 & 56 \\
- Ocupación Vocacional & 5 & 3 \\
Principal sostén en el hogar & & \\
- Sí & 35 & 35.7 \\
- No & 63 & 64.3
\end{tabular}




\begin{tabular}{lcc}
\hline \multicolumn{1}{c}{ Característica } & $f$ & $\%$ \\
\hline Grado de Obesidad & & \\
- Obesidad grado I & 29 & 29.6 \\
- Obesidad grado II & 38 & 38.8 \\
- Obesidad grado III & 26 & 26.5 \\
- Obesidad de grado IV & 5 & 5.1 \\
Diagnóstico de obesidad & & \\
- Sí & 83 & 84.7 \\
- No & 15 & 15.3 \\
Obesidad en familiares & & \\
- Sí & 55 & 56.1 \\
- No & 43 & 43.9 \\
Tratamiento de obesidad & & \\
- Sí & 88 & 89.8 \\
- No & 10 & 10.2 \\
\hline M = media; f frecuencia & &
\end{tabular}

Instrumentos

Se aplicó un cuestionario de condiciones sociodemográficas, la adaptación al español de Conde y Useros del Inventario de Depresión BDI-II de Beck, Steer y Brown (1996), y la validación cubana de Spielberg y Díaz Guerrero (1975) del Inventario de Ansiedad Rasgo-Estado (STAI) de Spielberg, Gorsuch y Lushene (1970).

El BDI-II consta 21 ítems, cada reactivo contiene $4 \circ 5$ frases y se le pide a la persona que seleccione aquellas que a su sentir le parecen más aplicables a su estado. La confiabilidad de alfa de Cronbach es variable entre .53 y .93 , así como una confiabilidad test-retest de .75. En esta investigación se obtuvo un coeficiente de coeficiente de confiabilidad alfa de Cronbach de .753 en el BDI. Cuenta una validez general para ser usado como herramienta para el diagnóstico de depresión mayor según DSM-IV (Sanz \& Vázquez, 1998). Su validez concurrente varía entre .62 y .77 al correlacionar con evaluaciones clínicas (reating scales) y entre $.41 \mathrm{y} .83 \mathrm{al}$ correlacionar con las escalas de Hamilton (Hrs/d), Hathaway y MacKinley (MMPI-d), Zung (SDS) y Lubding (Dacl) (López \& Polo, 2002).

EI STAI (o IDARE, por sus siglas en español) consta de 40 ítems. Está constituido por dos escalas de autoevaluación para medir dos dimensiones de la ansiedad: Ansiedad Rasgo y Ansiedad Estado. Ambos constan de 20 reactivos cada uno. Ansiedad Rasgo se define como las diferencias entre las personas en la tendencia a responder a situaciones percibidas como amenazantes, es decir, son disposiciones que permanecen latentes hasta que las señales de una situación las activa. Ansiedad Estado se define como una condición o estado emocional transitorio del organismo humano, caracterizada por sentimientos de tensión y de aprehensión subjetiva, conscientemente percibidos, y por un aumento de la actividad del sistema nervioso autónomo; los estados de ansiedad pueden variar en intensidad y fluctuar a través del tiempo (Estrada, Gante \& Hernández, 2008). Se valoró en una escala de cuatro categorías. Para la escala de Ansiedad Rasgo fueron: (a) Casi nunca, (b) Algunas veces, (c) Frecuentemente y (d) Casi siempre. Para la escala Ansiedad Estado fueron: (a) No en lo absoluto, (b) Un poco, (c) Bastante y (d) Mucho. Buela y Guillén (2011) la estabilidad de las puntuaciones o el índice de fiabilidad alfa de Cronbach fue 0.90 para Ansiedad Rasgo y 0.94 para Ansiedad Estado. En esta investigación, los coeficientes de confiabilidad alfa de Cronbach fueron de .87 para Ansiedad Rasgo y .82 para Ansiedad Estado. El STAI presenta validez de criterio por correlacionar significativamente con el Inventario Multifásico de la Personalidad de Minnesota, con el Edwards Personal Preference Schedule y el Mooney Problem Check List. Por lo expuesto, se garantiza la validez del inventario (López \& Polo, 2002).

\section{Procedimiento}

En una primera fase, se realizó la selección de la muestra de acuerdo a los criterios de inclusión y exclusión. Posteriormente se presentaron los procedimientos, los instrumentos de investigación, las consideraciones éticas y las informaciones 
generales de la investigación. Una vez obtenido el consentimiento informado de los participantes seleccionados, se administraron los instrumentos de forma individual y autoadministrada teniendo una duración aproximada entre 40 y 50 minutos cada una. La aplicación estuvo a cargo de las investigadoras de este estudio.

\section{Consideraciones éticas}

En el estudio se consideró el código de ética en las investigaciones con sujetos humanos y se les presentó a los participantes una carta informativa con estas consideraciones. El propósito de la carta de consentimiento informado fue documentar el consentimiento de la persona para participar de la investigación presentándosele información para que pueda tomar una decisión de forma voluntaria. Se le presentó el objetivo de la investigación, el tiempo aproximado que tomó responder los cuestionarios, además de expresarles que la participación fue voluntaria, garantizando anonimato $y$ confidencialidad, se permitió hacer preguntas así como retirarse en cualquier momento de la aplicación. No se puso en riesgo a las personas y la explicación de la investigación se realizó en el nivel del lenguaje de los sujetos para una compresión correcta de la información.

\section{Procedimientos estadísticos}

Los análisis estadísticos se realizaron con el programa SPSS para Windows (versión 17). Se calcularon estadísticos descriptivos para los datos sociodemográficos y las puntuaciones obtenidas en el BDI y el STAI. Con la prueba Kolmogorov-Smirnov se verificó que la distribución de las puntuaciones seguía la curva de normalidad, lo cual determinó el empleo de pruebas paramétricas para el análisis correlacional y la comparación entre grupos. Con la prueba $t$ de Student y ANOVA se compararon las medias de las puntuaciones obtenidas en el BDI y en el STAI, y se correlacionaron con el coeficiente de correlación $r$ de Pearson.

\section{RESULTADOS}

En la Tabla 2 se presentan las medias (M) desviaciones estándar (DE) de las puntuaciones obtenidas en el BDI y en el STAI, tanto de la muestra total como de las correspondientes a cada grupo según las variables sociodemográficas estudiadas.

En Ansiedad-Estado, se encontró diferencia al comparar las medias de las puntuaciones de quienes se encontraban recibiendo tratamiento psicológico, nutricionista y médico, con las de quienes no recibían dicho tratamiento $(t=-5.56$; $p=.000$ ), donde la media de los que no recibian tratamiento fue significativamente más alta. En Ansiedad-Rasgo, se encontró diferencia entre hombres y mujeres ( $t=-3.46$; $p=.001$ ), donde la media de las mujeres fue significativamente mayor que la de los hombres. En Depresión, la media de las mujeres fue significativamente más alta que la de los hombres $(t=-2.31 ; p=.023)$, al igual que la media del grupo de menores de 30 la cual fue significativamente mayor que la de 30 años o más ( $t=2.63 ; p=.010)$. Igualmente, fueron significativamente mayores las medias de quienes son principal sostén de su hogar por sobre quienes no $(t=-2.25$; $p=.027$ ), entre quienes refirieron tener diagnóstico de obesidad y quienes refirieron no tenerlo $(t=3.24 ; p=.002)$ y entre quienes se encontraban recibiendo tratamiento $y$ quienes no recibían dicho tratamiento $(t=2.21 ; p=.03)$.

Adicionalmente, se encontraron correlaciones positivas y estadísticamente significativas al correlacionar las puntuaciones obtenidas en STAI-Estado con STAI-Rasgo $(r=.544 ; p=.000)$, STAI-Estado con BDI ( $r=.417 ; p=.000)$, como también STAI-Rasgo con BDI $(r=.610 ; p=.000)$. 
TABLA 2.

Estadísticos descriptivos de puntuaciones en el STAI y en el BDI.

\begin{tabular}{|c|c|c|c|c|c|c|c|}
\hline & \multicolumn{2}{|c|}{ STAI-Estado } & \multicolumn{2}{|c|}{ STAI-Rasgo } & \multicolumn{2}{|c|}{$\mathrm{BDI}$} \\
\hline & & $M$ & $D E$ & $M$ & $D E$ & $M$ & $D E$ \\
\hline \multicolumn{2}{|c|}{ Puntuación total de la muestra } & 45.39 & 11.53 & 43.27 & 4.99 & 15.89 & 7.57 \\
\hline \multirow[t]{2}{*}{ Rango de edad } & Menores de 30 años & 45.42 & 11.34 & 43.88 & 4.49 & 17.80 & 7.28 \\
\hline & 30 años o más & 45.35 & 11.85 & 42.63 & 5.43 & 13.90 & 7.41 \\
\hline \multirow[t]{2}{*}{ Sexo } & Hombres & 40.84 & 10.74 & 39.89 & 3.84 & 12.37 & 6.47 \\
\hline & Mujeres & 46.48 & 11.51 & 44.08 & 4.91 & 16.73 & 7.60 \\
\hline \multirow[t]{4}{*}{ Estado civil } & Soltero/a & 45.02 & 11.45 & 43.66 & 4.94 & 17.39 & 7.48 \\
\hline & Casado/a - Unión de Hecho & 48.18 & 11.60 & 43.18 & 5.40 & 14.30 & 7.64 \\
\hline & Divorciado/a - Separado/a & 35.83 & 2.93 & 40.83 & 2.93 & 11.67 & 6.86 \\
\hline & Viudo/a & 40.67 & 15.04 & 41.67 & 4.51 & 13.67 & 4.93 \\
\hline \multirow[t]{6}{*}{ Nivel académico } & Primer Ciclo & 49.00 & 31.11 & 45.50 & 4.95 & 20.50 & 2.12 \\
\hline & Segundo Ciclo & 47.25 & 12.28 & 45.75 & 3.10 & 13.50 & 7.05 \\
\hline & Tercer Ciclo & 39.00 & 12.70 & 42.86 & 5.27 & 18.14 & 10.04 \\
\hline & Educación Media & 42.76 & 11.32 & 42.88 & 5.73 & 15.48 & 7.77 \\
\hline & Título Universitario & 46.62 & 10.81 & 42.93 & 4.82 & 15.49 & 7.59 \\
\hline & Ocupación Vocacional & 51.00 & 9.72 & 46.60 & 3.51 & 19.20 & 4.27 \\
\hline \multirow[t]{2}{*}{ Principal sostén } & Son el principal sostén & 42.34 & 11.37 & 42.74 & 4.34 & 13.63 & 7.92 \\
\hline & No son el principal sostén & 47.08 & 11.36 & 43.56 & 5.32 & 17.14 & 7.13 \\
\hline \multirow[t]{4}{*}{ Grado de obesidad } & Obesidad tipo I & 47.17 & 12.49 & 42.31 & 4.80 & 14.21 & 7.42 \\
\hline & Obesidad tipo II & 44.13 & 11.45 & 42.82 & 4.44 & 16.08 & 6.83 \\
\hline & Obesidad tipo III & 46.27 & 10.29 & 45.15 & 5.74 & 18.08 & 8.42 \\
\hline & Obesidad tipo IV & 40.00 & 13.34 & 42.40 & 4.56 & 12.80 & 8.04 \\
\hline \multirow{2}{*}{$\begin{array}{l}\text { Diagnóstico de } \\
\text { Obesidad }\end{array}$} & Con diagnóstico & 45.71 & 11.57 & 43.59 & 4.86 & 16.89 & 7.40 \\
\hline & Sin diagnóstico & 43.60 & 11.56 & 41.47 & 5.49 & 10.33 & 6.10 \\
\hline \multirow[t]{2}{*}{ Obesidad familiar } & Con familiares con obesidad & 45.65 & 10.93 & 43.28 & 5.01 & 15.35 & 7.11 \\
\hline & Sin familiares con obesidad & 45.18 & 12.08 & 43.25 & 5.02 & 16.31 & 7.95 \\
\hline \multirow{2}{*}{$\begin{array}{l}\text { En tratamiento de } \\
\text { obesidad }\end{array}$} & Sin tratamiento de obesidad & 46.89 & 10.96 & 43.27 & 4.97 & 15.33 & 7.17 \\
\hline & Con tratamiento de obesidad & 32.20 & 7.50 & 43.20 & 5.43 & 20.80 & 9.50 \\
\hline
\end{tabular}

\section{DISCUSIÓN}

Contrariamente a lo que se pudiese esperar, el grupo que se encontraba recibiendo tratamiento mostró una media mayor en síntomas depresivos. Los participantes que se encontraban en tratamiento, formaban parte de un programa televisivo de transmisión diaria que promueve principalmente el mejoramiento de la calidad de vida a través de una vida más saludable y el criterio de permanencia en el programa es el descenso del $10 \%$ de su peso de la semana anterior. Estas personas pudieron haber experimentado varios momentos de frustración y pena al intentar descender de peso y volviendo a subir posteriormente, hechos que pudieron precipitar la aparición de síntomas depresivos y estar reforzados por el miedo a volver a vivir una situación similar, por el miedo a ser eliminados del programa y por el temor a ser juzgados negativamente por los profesionales de salud (médicos, nutricionistas, psicólogos) encargados de su tratamiento. Reportes de investigación han afirmado que cuando una persona baja de peso y vuelven a subirlo, 
aumentan los niveles de frustración y pena, e inclusive que estas variables sufren un incremento por los mismos profesionales encargados del tratamiento quienes sugieren que el fracaso en el tratamiento se debe principalmente a la falta de ganas 0 esfuerzo para bajar de peso (SeijasBuschiazzo y Feuchtmann-Sáez, 1997).

Paralelamente, si bien se encuentran en tratamiento para la obesidad y otros trastornos físicos y psicológicos asociados, el IMC promedio de ambos grupos demuestra que existía un mayor grado de obesidad en el grupo con tratamiento, lo cual indicaría que cualquier trastorno (como la depresión) pudiera encontrarse mucho más arraigado por la cronicidad y gravedad de la obesidad, así como también ser consecuencia de otras patologías asociadas que presentan estas personas.

Luppino et al. (2010) demostraron la asociación entre obesidad y depresión, y afirmaron que así como la obesidad aumenta el riesgo de depresión (mayor para los clínicamente diagnosticados), la depresión es predictiva para el desarrollo de obesidad. Podría ampliarse la investigación para investigar los factores causales de obesidad en este grupo de personas que se encontraban en tratamiento, explorar el tiempo que se encuentran expuestos a síntomas depresivos y compararlos con el inicio y evolución de su obesidad.

Sería interesante determinar si las personas que participan del programa televisivo de descenso de peso hacen propio los objetivos planteados por el programa como sus principales motivadores para seguir en competencia 0 si existen otros factores. Podría investigarse si existe influencia de los estereotipos de delgadez socialmente aceptados y que son más promovidos por medios de comunicación como la televisión, tal como exponen en su investigación Jiménez-Cruz y Silva-Gutiérrez (2010). Estas investigadoras citan a varios investigadores quienes afirman que los medios de comunicación transmiten valores e ideales que las personas internalizan y que la delgadez (o aspecto físico como ideal sobrevalorado socialmente) es uno de los valores más poderosamente transmitido por dichos medios, poniendo en riesgo a trastornos de la conducta alimentaria. Así también, citan a otros investigadores quienes afirman que los medios de comunicación presentan imágenes de personas con cuerpos muy delgados, con peso inferior al considerado médicamente normal. En su investigación experimental, Jiménez-Cruz y Silva-Gutiérrez (2010) demostraron que las mujeres con riesgo de trastornos alimentarios presentaron niveles mayores de ansiedad y depresión en el postest tras la presentación de imágenes de mujeres delgadas y con obesidad, observándose efecto opuesto en el grupo sin riesgo.

Por el contrario, quienes no recibían tratamiento para la obesidad presentaron mayores puntuaciones media en ansiedadestado en relación con quienes recibían tratamiento, y no se encontró diferencias entre ambos grupos en ansiedad-rasgo. El estado es una condición transitoria del organismo que depende de una situación, en cambio, un rasgo es una característica relativamente permanente de la persona (Cohen \& Swerdlik, 2002). Este resultado menor de ansiedad-estado en quienes recibían tratamiento pudo deberse al tratamiento al cual estaban sometidos al momento de la evaluación.

Gómez-Peresmitré et al. (2006) también confirmaron los hallazgos en depresión y ansiedad encontrados al comparar las personas que se encontraban en tratamiento y quienes no. Citan a varios investigadores para afirmar que existen varios trastornos emocionales asociados con el tratamiento de la obesidad y menciona entre ellos a la depresión, la ansiedad, la irritabilidad y la preocupación por la comida. Sostienen que la obesidad 
está asociada a baja adherencia al tratamiento, evolución crónica y variedad en su etiología, lo cual dificulta más su cura, aumenta la recidiva y convirtiendo la intervención en un tratamiento paliativo.

Como se mencionó previamente, el hecho de que la obesidad aumenta el riesgo de depresión y que este riesgo es mayor para los clínicamente diagnosticados (Luppino et al., 2010) podría explicar que las puntuaciones media de síntomas depresivos sea mayor en quienes refirieron tener diagnóstico de obesidad que en quienes refirieron no tenerlo.

Tras comparar los menores de 30 años con los de 30 años o más, se encontró mayor puntuación de síntomas depresivos en los primeros. Esto pudo haberse dado ya que las personas en etapa de adultez temprana en relación a las de la adultez madura en adelante presentan menores índices de afrontamiento, resiliencia, capacidad de resolución de problemas y otros factores como la tolerancia a la frustración, entre otros. Arrivillaga Quintero et al. (2004) confirman esto al referirse que ciertos estresores específicos para los jóvenes y el déficit de estrategias de afrontamiento que les caracteriza, son un factor clave de predisposición y desencadenante de la depresión.

En esta investigación se encontró que las mujeres presentaron mayor puntuación media en relación a los hombres en síntomas depresivos y en ansiedad-rasgo. Hallazgo similar se dio en el estudio de Ríos Martínez et al. (2008) en donde las mujeres con obesidad también presentaron puntaje medio mayor en depresión y ansiedad. Arenas y Puigcerver (2009) citan a varios investigadores para expresar que los trastornos de ansiedad son más prevalentes en mujeres en edad reproductiva (aproximadamente 2 a 3 veces más). También sostienen que el sexo influye en la prevalencia de los trastornos mentales, en la manifestación y expresión de los síntomas, en la voluntad para solicitar asistencia profesional, en la evolución de la enfermedad $y$ en la respuesta al tratamiento. Por su parte, Franchi (2001) afirma que la prevalencia de depresión en mujeres es mayor que la presente en hombres en una relación dos a uno.

Arrivillaga Quintero et al. (2004) también afirman que la depresión es más frecuente en mujeres debido a factores sociales 0 psicológicos y que esta prevalencia es mayor porque ellas están más dispuestas a expresar o reportar su depresión. Expresan que podrían existir factores fisiológicos causantes de esta mayor prevalencia, como los cambios hormonales, estrés, funcionamiento de la glándula tiroides, entre otros.

La cantidad de trabajos que relacionan sexo 0 género con ansiedad aumentó considerablemente desde 1990 (Arenas \& Puigcerver, 2009), sin embargo, organizaciones como la Asociación Americana de Psicología, la Asociación Americana de Psiquiatría, el Instituto Nacional de Salud Mental y la Organización Mundial de la Salud afirman que todavía se necesitan muchas investigaciones para aclarar la relación entre género y psicopatología.

En este estudio se encontró que quienes no son el principal sostén de su hogar presentan niveles mayores de síntomas depresivos que aquellos que lo son. Una persona con obesidad, a consecuencia del estigma social al que está sometido, podría tener menores oportunidades laborales y esto llevar a la aparición de síntomas depresivos debido a la frustración que podrían experimentar en estas situaciones. Seijas Buschiazzo y Feuchtmann Sáez (1997) sostienen que la obesidad constituye uno de los principales estigmas sociales acompañándose de disminución de oportunidades de trabajo y educación con repercusión en el estado psicológico con la aparición de síntomas como ansiedad, 
afectos disfóricos, disminución del autoconcepto, de la autoimagen y de la autoeficacia, y el empeoramiento de las relaciones interpersonales. Ser el principal sostén del hogar implica un mayor grado de responsabilidad y de actividad de la persona para satisfacer sus necesidades personales y de aquellas personas que se encuentran bajo su responsabilidad. El hecho de no tener dicha responsabilidad que implica ser el sostén de su hogar, sumado a su baja actividad laboral por las pocas posibilidades de empleo debido al estigma social que recibe a causa de su obesidad, podrían ser reforzadores de la aparición de síntomas depresivos.

Existen varias investigaciones que estudiaron la correlación entre ansiedad y depresión. Estos estudios encontraron correlaciones positivas y estadísticamente significativas entre ambas variables, al igual que lo reportado en esta investigación. Las correlaciones positivas indican que a mayor ansiedad se presenta mayor nivel de depresión (y viceversa) así como una disminución paralela en el nivel de ambas variables. Canto \& Castro (2004), Estrada, Gante \& Hernández (2008), Agudelo, Casadiegos \& Sánchez (2009), Méndez et al. (2010) y Ramírez y Hernández (2012) encontraron correlaciones positivas y significativas entre Depresión y AnsiedadRasgo y entre Depresión con AnsiedadEstado, tal como ocurrió en la presente investigación.

Las puntuaciones obtenidas en esta investigación con la correlación de Pearson entre STAI Estado, STAI Rasgo y BDI, coinciden con los resultados de la investigación de Calderón, Forns y Varela (2010b). Estos investigadores concluyeron que los trastornos de alimentación, como lo es la obesidad, son explicadas por la mayor presencia de síntomas de ansiedad y de depresión.

En conclusión, esta investigación encontró correlaciones positivas entre ansiedad y depresión, lo que demuestra la estrecha relación existente entre las variables e indica que dichos constructos varían en el mismo sentido.

\section{REFERENCIAS}

Álvarez, J., Monereo, S. \& Moreno, B. (2000). Obesidad: epidemia del siglo XXI. Madrid, España: Editorial Díaz de Santos. Recuperado de http://books.google.es/books?id=mLj0qJkQ $3 \mathrm{fwC} \&$ printsec=frontcover\&hl=es\&source= $g b s \_a t b \# v=o n e p a g e \& q \& f=f a l s e$

Arenas, M.C., \& Puigcerver, A. (2009). Diferencias entre hombres y mujeres en los trastornos de ansiedad: una aproximación psicobiológica. Escritos de Psicología, 3(1), 20-29. Recuperado de http://www.redalyc.org/pdf/2710/27102040 3003.pdf

Arrivillaga Quintero, M., Cortés García, C., Goicochea Jiménez, V.L., \& Lozano Ortiz, T.M. (2004). Caracterización de la depresión en jóvenes universitarios. Universitas Psychologica, 3(1), 17-26.

Ayela, M. (2009). Obesidad: problemas y soluciones. Alicante, España: Editorial Club Universitario. Recuperado de http://www.editorial-clubuniversitario.es/pdf/3575.pdf

Beck, A. T., Brown, G. K. \& Steer, R. A. (1996). BDI-II. Beck Depression Inventor Manual. (2da ed.). San Antonio, TX: The Psychological Corporation.

Buela, G. \& Guillén, A. (2011). Actualización psicométrica y funcionamiento diferencial de los ítems en el State Trait Anxiety Inventory (STAI). Revista Psicothema 23(3), 510-515.

Bobadilla-Suárez, S., y López-Avila, A. (2014). Distorsión de imagen corporal percibida e imaginada. Un posible factor para la obesidad y el sobrepeso en mexicanos. Revista Médica del Instituto Mexicano del Seguro Social, 52(4), 408-414. 
Calderón, C., Forns, M., \& Varea, V. (2010a). Implicación de la ansiedad y la depresión en los trastornos de alimentación de jóvenes con obesidad. Nutrición Hospitalaria, 25(4), 641-647.

Calderón, C., Forns, M., \& Varea, V. (2010b). Obesidad infantil: ansiedad y síntomas cognitivos y conductuales propios de los trastornos de alimentación. Revista Anales de Pediatría, 71(6), 489-494.

Cárdenas, K., Ontiveros, C., \& Villaseñor, S., (2006). Salud Mental y Obesidad. Revista Médica Latinoamericana, 8(2), 86-90.

Castillo, A., García, M. \& Guzmán, M. (2010). Factores Psicosociales relacionados con la obesidad (Trabajo de investigación). Universidad Autónoma del Estado de Hidalgo, Pachuca de Soto, México. Recuperado

de http://www.uaeh.edu.mx/investigacion/icsa/ LI_Evalulnter/Meli_Garcia/8.pdf

Cohen, R. J., \& Swerdlik, M. E. (2002). Pruebas y evaluación psicológicas. Introducción a las pruebas y a la medición. México D.F., México: McGraw-Hill.

Cruz Sánchez, M., Tuñón Pablos, E., Villaseñor Farías, M., Álvarez Gordillo, G.C., \& Nigh Nielsen, R.B. (2013). Sobrepeso y obesidad: una propuesta de abordaje desde la sociología. Región y sociedad, 25(57), 165-202.

Días, D., \& Enríquez, D. (2007). Obesidad Infantil, Ansiedad y Familia. Boletín Clínico Hospital Infantil del Estado de Sonora, 24(1), 22-26.

Estrada, G., Gante, J., \& Hernández, A. (2008). Niveles de ansiedad y depresión en personas con sobrepeso y obesidad (Trabajo de investigación) Universidad del Valle de México, Mexico D.F., México. Recuperado de https://es.scribd.com/doc/258090597/Ansie dad-mave\#download

Franchi, S. (2001). La depresión en el hombre. Interdisciplinaria, 18(2), 135-154.

Gómez-Peresmitré, G., González de Cossío, M., Sois Torres, C., Cuevas-Renaud, C., Unikel Santoncini, C., Saucedo Molina, T., \& Rodríguez de Elías, R. (2006). Obesidad en población universitaria: Prevalencia y relación con agresión y conductas compensatorias y alimentarías de riesgo. Revista Mexicana de Psicología, 23(2), 135-147.
Jiménez-Cruz, B.E., \& Silva-Gutiérrez, C. (2010). Riesgo para trastorno alimentario, ansiedad, depresión y otras emociones asociadas a la exposición de imágenes publicitarias. Anales de Psicología, 26(1), 11-17.

López, A., \& Polo, V. (2002). Estudio descriptivo sobre las alteraciones psicológicas en un grupo de víctima del desplazamiento forzoso en la ciudad de Bogotá (Tesis de grado). Universidad de la Sabana, Chía, Colombia. Recuperado de http://intellectum.unisabana.edu.co/bitstrea $\mathrm{m} / \mathrm{handle} / 10818 / 4070 / 131269 . \mathrm{pdf}$

Luppino, F.S., De Wit, L.M., Bouvy, P.F., Stijnen, T., Cuijpers, P., Penninx, B.W., \& Zitman, F.G. (2010). Overweight, obesity, and depression: a systematic review and meta-analysis of longitudinal studies. Archives of general psychiatry, 67(3), 220229.

OMS (2012). Temas de salud: Obesidad. Recuperado de http://www.who.int/mediacentre/factsheets/ fs311/es/

OMS (2014). Centro de Prensa: Obesidad y sobrepeso. Recuperado el 25 de Mayo de 2015, de http://www.who.int/mediacentre/factsheets/ fs311/es/

Pompa, E. (2011). Evaluación de un Programa Multidisciplinario para Reducir los Niveles de Ansiedad, Depresión e Índice de Masa Corporal en Niños con Sobrepeso y Obesidad (Tesis Doctoral). Universidad Autónoma de Nuevo León, Nuevo León, México. Recuperado de http://eprints.uanl.mx/2818/1/Tesis_Origina I.pdf.

Ríos Martínez, B., Rangel Rodríguez, G., Álvarez Cordero, R., Castillo González, F., Ramírez Wiella, G., Pantoja Millán, J., Yáñez Macías-Valdez, B., Arrieta Maturino, E., \& Ruiz Ochoa, K., (2008). Ansiedad, depresión y calidad de vida en el paciente obeso. Revista Médica Latinoamericana, 6(4), 147-153.

Rubio, M. A. (2012). Estudio de la Relación entre Ansiedad y Obesidad a través del Cuestionario de Ansiedad de Estado Rasgo (STAl) valoración del Perfil Dietético y Psiconutricional (Tesis para optar el Máster en Medicina Cosmética y del Envejecimiento). Universidad Autónoma de Barcelona, Barcelona, España. 
Recuperado de http://www.semcc.com/master/files/Obesid ad\%20y\%20ansiedad\%20\%20Dr.\%20Rubio.pdf

Sanz, J., \& Vázquez, C. (1998). Fiabilidad, Validez y Normativos del Inventario para la Depresión de Beck. Revista Psicothema, 10(2), 303-318.

Seijas Buschiazzo, D., \& Feuchtmann Sáez, C. (1997). Obesidad: factores psiquiátricos y psicológicos. Boletín Escuela de Medicina de la Pontificia Universidad Católica de Chile, 26, 38-41.

Silvestri, E. (2005). Aspectos Psicológicos de la Obesidad. Importancia de su identificación dentro de un enfoque interdisciplinario (Tesis de posgrado en obesidad para optar al título de doctor de nutrición). Universidad Favaloro, Córdoba, Argentina. Recuperado de http://www.nutrinfo.com/biblioteca/monogra fias/ob05-02.pdf

Spielberger, C. D., Gorsuch, R. L., \& Lushene, R. (1970). Manual for the State-Trait Anxiety Inventory. Palo Alto, CA: Consulting Psychologists Press.

Spielberg C. D., \& Díaz-Guerrero, R. (1975). Inventario de Ansiedad Rasgo-Estado (IDARE). México D.F., México: El Manual Moderno. 